\title{
Montoya Huamaní, Segundo (2018). Conflictos de interpretación en torno al marxismo de Mariátegui. Lima: Heraldos Editores.
}

\begin{abstract}
Miraría con desdén a los repetidores mediocres de sus frases. Amaría solo una juventud capaz de traducir en acto lo que en él no pudo ser sino idea y no se sentiría renovado y renacido sino en hombres que supieran decir una palabra verdaderamente nueva, verdaderamente actual.
\end{abstract}

José Carlos Mariátegui.

Wittgenstein decía que un libro solo podía ser comprendido por aquellos que previamente hubieran pensado las mismas ideas en él contenidas; el libro de Segundo Montoya no es la excepción, sino la confirmación de una extraña y afortunada sintonía entre la lectura que Mariátegui hizo de la realidad peruana y la relectura que el autor hace de su pensamiento y de los Conflictos de interpretación de su marxismo después de 90 años. Puedo prever la indignación y rabia de un sector de la izquierda dura y ortodoxa que todavía busca afiliar a Mariátegui a un "sendero luminoso" totalmente oscuro y dogmático. Mariátegui afirmaba en sus 7 ensayos: "[...] no somos un pueblo que asimila las ideas y los hombres de otras naciones, impregnándolas de su sentimiento y su ambiente"; consciente de este problema, el marxismo en Mariátegui fue pensado, sentido y vivido a partir de nuestra realidad, la semilla marxista fue sembrada en tierra latinoamericana y produjo frutos fecundos.

Conflictos de interpretación en torno al marxismo de Mariátegui es una obra potente y "liminar", porque representa una nueva "entrada" al marxismo del Amauta - como bien lo señala Miguel Mazzeo en el prólogoEste libro desarrolla un "itinerario" de autodefinición personal, es decir, el autor busca superar su "marxismo emocional" (o en clave Edad de Piedra en clave mariateguina) y asume el reto de filosofar "desde la periferia de la periferia", releer el marxismo de Mariátegui desde nuevas "premisas epistemológicas". 
Contra aquellos filósofos latinoamericanos que aún optan por filosofar desde una perspectiva eurocéntrica - pensar de espaldas a su realidad- y constituirse sin ser conscientes de ello en "elegantes flores de invernadero académico", nuestro autor se inserta deliberadamente en la tradición filosófica latinoamericana y busca dialogar con ella, para enriquecerla.

El primer capítulo, "El marxismo abierto como coordenada teórica”, está compuesto por tres ensayos: (1) "Augusto Salazar Bondy: El marxismo abierto de Mariátegui"; (2) "David Sobrevilla: Lo vivo y lo muerto del pensamiento de Mariátegui”; y, (3) “José Ignacio López Soria: 'Adiós a Mariátegui””, como intento de repensar el Perú en clave posmoderna. En el primer ensayo el autor plantea que la interpretación salazariana del pensamiento de Mariátegui como "marxismo abierto" será el punto de partida de sus reflexiones, su coordenada teórica en la búsqueda de una noción articuladora que permita realizar un "cambio de Gestalt" en las interpretaciones sobre Mariátegui, desde el punto de vista de los autores de la "inflexión decolonial" (o grupo modernidad/colonialidad: Quijano, Dussel, Mignolo, Lander, Castro, entre otros). El autor hace hincapié en la necesidad de una lectura crítico-hermenéutica de nuestros filósofos, que no se limite a ser una mera exposición descriptiva de sus ideas, sino que sea un constante diálogo y confrontación de ideas que permita ir con los filósofos más allá de ellos. Luego de realizar una crítica a algunos planteamientos erróneos presentes en dos publicaciones sobre Salazar Bondy del año 2013 —el libro El concepto de alienación según Augusto Salazar Bondy de Andrés Espíritu y el artículo "Augusto Salazar Bondy. El esplendor, ocaso y fracaso del filosofar marxista" de Fernando Muñoz Cabrejo-, Segundo Montoya analiza la noción de "marxismo abierto" según Salazar Bondy. Así, sostiene que Mariátegui distingue entre dos tipos de revisionismo: (1) revisionismo verdadero o fecundo que consiste en una crítica fundada y renovadora; (2) revisionismo falso o estéril que consiste en una crítica falaz y regresiva. Citando a Mazzeo, resalta la noción de "dogmabrújula" y sostiene que "Mariátegui concibe el dogma como la doctrina que debe ser constantemente fecundada y actualizada creativamente en forma colectiva" (p. 32). Establece, asimismo, una analogía entre el marxismo de Mariátegui y los programas de investigación científica de Imre Lakatos, además de considerar que 
existe una "matriz básica" o "centro firme" en el marxismo de Mariátegui y un "cinturón de seguridad" que incorpora elementos teóricos ajenos al pensamiento marxista como son "el mito soreliano, la metafísica bergsoniana, el pragmatismo jamesiano, el psicoanálisis freudiano, el voluntarismo nietzscheano, etcétera" (p. 35). Nuestro autor cita un conjunto de intelectuales que concuerdan con la interpretación salazariana: José María Aricó, "el marxismo solo podía ser creador a condición de mantener abiertos los vasos comunicantes con la cultura contemporánea"; Raúl Fornet-Betancourt, "defender el marxismo como un método de interpretación es defender al mismo tiempo un marxismo teóricamente abierto hacia adelante"; Nestor Kohan, "el carácter abierto del marxismo de Mariátegui le permitió [...] incorporar a sus corpus teórico lo más avanzado de aquellos "radicales" años 20"; Pablo Guadarrama, "uno de los primeros rasgos que emancipó al marxismo de Mariátegui del marxismo ortodoxo, fue su actitud ampliamente receptiva en relación con las nuevas corrientes contemporáneas" y, por último, Antonio Mellis, quien sostiene que dicha "actitud abierta" sería interpretada por los marxistas ortodoxos como un acto de herejía, y burdo revisionismo. Luego se analizan las críticas planteadas a la noción salazariana del "marxismo abierto" por Adalbert Dessau, quien considera más adecuado el adjetivo de "dialéctico" en vez de "abierto"; Zenón Depaz, quien reivindica la heterodoxia del Amauta, pero rechaza la noción de un "marxismo abierto"; Aníbal Quijano (en la década de 1980), quien rechaza la noción de "marxismo abierto" por considerar el socialismo humanista de Salazar como una doctrina reaccionaria y ajena al socialismo revolucionario. El autor concluye señalando que una posible causa del rechazo de la noción "marxismo abierto" de Salazar se basó fundamentalmente en que era una interpretación políticamente incorrecta para el "orden del discurso" impuesto por el marxismo-soviético-chino de aquellos años.

Luego, el autor reflexiona sobre los análisis de David Sobrevilla y José Ignacio López Soria a los que caracteriza como "liquidacionistas". Con respecto a Sobrevilla, que busca distinguir entre lo vivo y lo muerto del pensamiento de Mariátegui, nuestro autor argumenta contra dos apreciaciones críticas realizada al Amauta: (1) su partidismo (falta de objetividad e imparcialidad) en cuanto al conocimiento y (2) su determinismo económico. Con respecto al primer punto, 
hace notar que en las Ciencias Sociales, la "objetividad" e "imparcialidad" son nociones problemáticas, y al respecto afirma: “[...] el partidismo del Amauta es un criterio político-epistemológico de análisis crítico, interpretación y producción de sus ideas, que atraviesan todos sus escritos" (p. 50); con respecto a la segunda crítica, el autor evidencia el error de atribuir un "determinismo económico" al Amauta, siguiendo a Dussel, considera que las tesis de Marx, más que deterministas son parte de una política realista y están inspiradas en motivaciones éticas. En su “Adiós a Mariátegui”, López Soria al parecer considera — desde una lectura en clave posmoderna - que el marxismo de Mariátegui se inserta dentro del proyecto moderno y, por ende, es una variedad del metarrelato marxista que ha perdido vigencia con la caída del socialismo real. Pero el autor acusa a López Soria de adoptar el "posmodernismo" de manera acrítica y descontextualizada, pues asume un "discurso filosófico" ajeno a nuestra realidad social, donde encontramos muchos rezagos premodernos y modernos que desembocan en una visión eurocéntrica, que no toma en cuenta la dialéctica entre centro-periferia propia de la modernidad.

El segundo capítulo denominado "Marxismo no-eurocéntrico en Mariátegui" se compone de tres ensayos: (1) "Raúl Fornet-Betancourt: un marxismo 'abierto' y 'des-centrado'”; (2) “Enrique Dussel: 'Intuiciones' de Mariátegui por desarrollar”; y (3) “Aníbal Quijano, improntas de Mariátegui en la colonialidad del poder".

En el ensayo, "Raúl Fornet-Betancourt: un marxismo 'abierto' y 'descentrado", el Segundo Montoya reflexiona y toma como fuente la Historia del marxismo en América Latina del filósofo cubano-alemán. El autor considera que la interpretación de Fornet-Betancourt resalta el descentramiento del marxismo eurocéntrico y su "naturalización" latinoamericana y su "apertura teórica" permitirá la asimilación de formas de pensamiento ajenas al marxismo y con ello "la ampliación del horizonte categorial del marxismo". En el ensayo “Enrique Dussel: 'intuiciones' de Mariátegui por desarrollar” describe cómo Dussel —utilizando los adjetivos que el mismo Mariátegui atribuye a Sorel— califica el marxismo de Mariátegui como una "filosofía de la revolución" impregnada de un "realismo psicológico y sociológico"; así, en él la realidad 
tiene primacía sobre la teoría, el mito predomina sobre la racionalidad abstracta, el mundo cultural del trabajador es antes que la materia, el indigenismo previo a la abstracta lucha proletaria europea, los sindicatos precursores del partido, y concluye: "Mariátegui no teme la heterodoxia, odia el dogmatismo" (p. 85). El autor aclara que Mariátegui se distancia de aquellos que asumen el dogma como un "itinerario" (verdad acabada), pero no de aquellos que asumen el dogma como una "brújula" (verdad procesual). El pensamiento mariateguino opta por situarse entre conceptos aparentemente opuestos e intenta mostrarnos la tensión y fecundidad que existe en su interacción y dinamismo; por ello, afirma el carácter fecundo de ciertas heterodoxias que enriquecen la tradición, el carácter fecundo de ciertas herejías que revitalizan el dogma. Dussel considera que el marxismo de Marx y Mariátegui implica un cuestionamiento ético, un rechazo al cientificismo y determinismo marxista. El marxismo de Mariátegui que intenta pensar el Perú a partir del "problema del indio" es coherentemente marxista con un capitalismo periférico, latifundista y preindustrial. La propuesta de Dussel es que se deben desarrollar en el plano teórico las categorías o "intuiciones" de Mariátegui (clase, etnia, pueblo, nación, etc.). Las comunidades indígenas no constituyen una clase social, ni naciones-Estado, sino etnias o naciones originarias, anteriores a los estados criollos mestizos del capitalismo dependiente, que se han resistido a ser subsumidas estrictamente como clase campesina. Para Dussel, la vigencia del pensamiento de Mariátegui radica en comprender que el "problema del indio" sigue siendo un problema fundamental en algunos países andinos de América.

En el ensayo "Aníbal Quijano: improntas de Mariátegui en la colonialidad del poder", el autor considera que el pensamiento de Quijano logra ir con Mariátegui, más allá de Mariátegui; considera que "la colonialidad del poder" se ha constituido a partir de ciertas intuiciones o improntas mariateguinas. El autor sostiene que el análisis de Quijano se basa en tres ejes temáticos: (1) La crítica a la racionalidad eurocéntrica a través de la tensión entre "mito y logos"; (2) la tematización del concepto de "raza" como base epistémica de poder colonial en lugar del concepto de "clase social" de origen marxista ortodoxo europeo; y (3) la "heterogeneidad histórico-estructural" de los múltiples "modos de producción" que operan articulados dentro de una "totalidad" en la economía 
peruana, acompañado de un análisis cronológico de los estudios de Quijano sobre Mariátegui. En la primera parte de este ensayo el autor analiza la propuesta de Quijano realizada en 1981. Esta engloba cuatro "intentos fallidos de recuperación mistificadora" que se han llevado a cabo sobre el pensamiento de Mariátegui, el autor se centra en su crítica a la noción de "marxismo abierto" de Salazar. Luego, entre los años 1986 y 1991, el autor analiza como la re-lectura de Mariátegui lleva a Quijano a pensar en una racionalidad alternativa a la modernidad eurocéntrica, a partir de la tensión entre las perspectivas cognoscitivas del mito y logos, que tiene sus raíces en las relaciones de poder coloniales surgidas con el descubrimiento de América. La imposibilidad de estudiar la especificidad ontohistórica de Latinoamérica, solo con el andamiaje epistemológico y metodológico de origen eurocentrista, llevará a Quijano a desarrollar su tesis de la "colonialidad del poder". Ya en 1991 sostiene que la singularidad del pensamiento de Mariátegui radica en la redefinición de las categorías marxistas desde una perspectiva distinta a la que dominaba entre los marxistas europeos y en el estalinismo internacional; para el autor, en Mariátegui no hay una aplicación mecánica del marxismo a la realidad peruana, sino una especie de naturalización o descentramiento del marxismo eurocéntrico, que en Haya de la Torre aparece como una "actitud", pero en Mariátegui constituye una "perspectiva epistemológica" alternativa y latinoamericana. El autor considera que la noción de "heterogeneidad estructural" de Quijano se articula a partir de una "impronta" mariateguina, esta noción posee dos aspectos: en primer lugar se opone al carácter homogéneo de una formación social y afirma la existencia de diversos "modos de producción" que se articulan y se oponen dentro de una totalidad social y en segundo lugar se opone también a la idea de que su configuración obedezca a una lógica unidireccional, universal e invariante, sino a partir de lógicas continuas y discontinuas de desarrollo. Ya en 1992, Quijano define su noción de colonialidad como una estructura de poder mundial con dos ejes: (1) el económico que se basa en la articulación de nuevas y diversas relaciones de producción y (2) el cultural donde se afirma la emergencia de nuevas identidades (indios, negros, blancos, mestizos). Quijano sostiene que el racismo y el etnicismo se convertirán en el fundamento de las relaciones de poder entre Europa y América, tras el descubrimiento de América. El poder de los colonizadores sobre los pueblos colonizados se extiende hacia una 
“colonización del imaginario", que llevó a los dominados a percibirse a sí mismos desde la mirada del colonizador. La racionalidad modernidad eurocéntrica se establece negando a los pueblos colonizados todo lugar y papel que no sean el de sometimiento, en la producción y desarrollo de la racionalidad. Para el autor, las intuiciones mariateguinas sobre la "raza" son incorporadas por Quijano a su perspectiva crítica de la colonialidad; por ello, en la nueva estructura global de control, "la raza" y "la división del trabajo" quedaron estructuralmente asociadas, reforzándose mutuamente. Por último, se analizan las relaciones entre "raza" y "nación" y como se comprendieron dichas relaciones en otros países, donde la búsqueda de una población nacionalmente homogénea produjo el exterminio de la raza indígena. Repensar estas categorías desde la dominación cultural es uno de los retos de la tradición intelectual latinoamericana.

El tercer capítulo denominado "Marxismo abierto y des-centrado en Mariátegui”" se compone de tres ensayos: (1) "Cuestiones abiertas en torno al marxismo de Mariátegui"; (2) "Límites, alcances y ambigüedades de la noción de 'apertura' en el marxismo de Mariátegui"; y (3) "Noción de 'epistemología crítica' o 'epistemología des-centrada' en el marxismo de Mariátegui”.

En "Cuestiones abiertas en torno al marxismo de Mariátegui" el autor analiza su forma de entender y de hacer la filosofía; considera que la actividad filosófica se inserta dentro de una tradición, y, como tal, debemos realizar la recuperación de nuestra tradición a partir del diálogo y debate de las ideas. El autor vuelve a Mariátegui pero no para elevar a dogma políticamente correcto sus ideas o propuestas de inicios del siglo XX, sino para descubrir aquellas influencias, intuiciones e improntas que poseen la fecundidad necesaria para repensar nuestra realidad del siglo XXI. Considera que una verdadera relectura del Amauta debe buscar “ir con Mariátegui más allá de Mariátegui”, y afirma que la filosofía de la liberación de Dussel y la colonialidad del poder son dignas herederas del legado de Mariátegui. De esta forma, busca "posicionar y normalizar la idea de que la filosofía es un ejercicio dialógico, crítico y, sobre todo, propositivo" (p. 129). Citando a Jean Grondin señala: "El hombre culto no es aquel que tiene una respuesta para todo, si no aquel que sabe plantear cuestiones y dejarlas abiertas, y muestra así que tiene un horizonte" (p. 127). 
En "Límites, alcances y ambigüedades de la noción de 'apertura' en el marxismo de Mariátegui", el autor observa que no obstante la palabra "apertura" no se encuentra presente en la obra de Mariátegui, sino la categoría de asimilación, dicho concepto se refiere al proceso teórico de incorporación de corrientes posteriores a Marx (voluntarismo, pragmatismo, espiritualismo, psicoanálisis, etcétera). No hay asimilación sin apertura; en Mariátegui aparece una actitud de apertura hacia ideas no marxistas, pero útiles a la causa revolucionaria. El autor equipara el marxismo de Mariátegui con un "programa de investigación" no en su contenido, sino en su forma lógica. Existe un "núcleo fuerte" que conforman los "rasgos esenciales" como son "la posición de clase o criterio partidista, el materialismo histórico como método, la revolución social, el análisis de la sociedad sobre la base de los heterogéneos modos de producción y la lucha de clases, entre otros. Y también con un "cinturón de seguridad" donde encontramos "el voluntarismo de Nietzsche, la moral de los productores de Sorel, el pragmatismo de James, el intuicionismo de Bergson y el psicoanálisis de Freud. Por ello, al "aperturarse" no pierde su carácter revolucionario y tampoco deriva en un revisionismo ingenuo.

En "Noción de 'epistemología crítica' o 'epistemología des-centrada' en el marxismo de Mariátegui”, el autor realiza un análisis del término "epistemología" de carácter aparentemente neutral para mostrarnos su "carácter ideológico". Desde un análisis sociológico de la producción y validación de los saberes científicos, siguiendo a Walter Mignolo, Castro Gómez y Sousa Santos, establece que Europa es el lugar "geopolíticamente" clave de validación de los saberes y discursos. De esta manera, existe un "[...] falaz consenso universal por parte de los especialistas y las instituciones que los respaldan para imponer su perspectiva y convertirla en una 'epistemología' hegemónica e imperial denominada eurocentrismo" (p. 135). El autor propone el uso del término "epistemología" pero busca dotarlo de un nuevo significado; para ello propone la categoría filosófica de "epistemología crítica", que busca oponerse a una epistemología eurocéntrica que invisibiliza su carácter ideológico y legitima desde Europa-centro la producción y validación de saberes de acuerdo con sus intereses hegemónicos y se impone en las periferias. Obviamente, dicha categoría tiene sus antecedentes en otros autores como Boaventura Sousa ("epistemología del sur"), 
Walter Mignolo (“epistemología otra") y Aníbal Quijano (“colonialidad de podersaber"). El autor cita a Cesar Germaná, quien establece algunas relaciones entre el pensamiento de Mariátegui y Quijano, destacando cómo la "vigorosa autonomía intelectual del Amauta" le permitió desarrollar "una perspectiva epistemológica enfrentada al eurocentrismo". El autor considera que la teoría de la colonialidad del poder de Quijano se ha constituido a partir de una relectura crítica de la obra y las intuiciones de Mariátegui. Frente a una universalidad eurocéntrica, el autor propone la pluriversalidad que consiste "[...] en una perspectiva que apuesta por visibilizar y hacer visibles la multiplicidad de conocimientos, formas de ser, y aspiraciones sobre el mundo" (p. 140).

Mientras que Salazar Bondy defendió la noción de "marxismo abierto" en Mariátegui, y Quijano interpreta al Amauta en función de una "racionalidad alternativa", Segundo Montoya nos propone la noción de "apertura epistemológico crítica" como nueva categoría hermenéutica y epistémica para pensar el marxismo de Mariátegui. Así, distingue dos ejes fundamentales desde el cual poder interpretar a Mariátegui: (1) la "apertura epistemológica" que implica la ampliación del horizonte categorial del marxismo a partir del análisis de la realidad, que en el caso de la realidad peruana implicó abordar el "problema del indio" y "la unidad tensional entre mito y logos" que se caracteriza por su "unimismamiento" o "pluriversalidad"; y (2) la "epistemología crítica", que sostiene que el pensamiento de Mariátegui se caracteriza por su "descentramiento" del eurocentrismo marxista y la constitución de un marxismo enraizado, re-creado, naturalizado desde Latinoamérica.

\section{Mirko Díaz Sánchez}

Universidad Nacional de Trujillo, Trujillo, Perú

Contacto:mjdsanz@gmail.com

https://orcid.org/0000-0003-0234-5769 